\title{
Diagonal reflection symmetries and universal four-zero texture
}

\section{Masaki J.S. Yang*}

Department of Physics, Saitama University,

Shimo-okubo, Sakura-ku, Saitama, 338-8570, Japan

E-mail: yang@krishna.th.phy.saitama-u.ac.jp

In this talk, we consider a set of new symmetries in the SM: diagonal reflection symmetries $R m_{u, v}^{*} R=m_{u, v}, m_{d, e}^{*}=m_{d, e}$ with $R=\operatorname{diag}(-1,1,1)$. By combining the symmetries with the four-zero texture $\left(m_{f}\right)_{11}=\left(m_{f}\right)_{13}=0$, the masses and mixing matrices of quarks and leptons are reproduced with precisions of $10^{-3}$. Since this scheme has only eight parameters in the lepton sector, it has four predictions; the Dirac phase $\delta_{C P} \simeq 203^{\circ}$, the Majorana phases $\left(\alpha_{2}, \alpha_{3}\right) \simeq\left(11.3^{\circ}, 7.54^{\circ}\right)$ up to $180^{\circ}$, and $m_{1} \simeq 2.5$ or $6.2 \mathrm{meV}$ with the normal hierarchy.

In this scheme, the type-I seesaw mechanism and a given neutrino Yukawa matrix $Y_{v}$ completely determine the structure of the right-handed neutrino mass matrix $M_{R}$. A $u-v$ unification predicts its masses to be $\left(M_{R 1}, M_{R 2}, M_{R 3}\right)=\left(O\left(10^{5}\right), O\left(10^{9}\right), O\left(10^{14}\right)\right) \mathrm{GeV}$ with a strong hierarchy $M_{R} \sim Y_{u}^{T} Y_{u}$.

The symmetries are approximately stable under the renormalization of SM. This statement holds without the four-zero texture as long as couplings in the first row and column of the Yukawa matrices are sufficiently small. Then, they can possess information on a high energy scale.

7th Symposium on Prospects in the Physics of Discrete Symmetries (DISCRETE 2020-2021)

29th November - 3rd December 2021

Bergen, Norway

\footnotetext{
${ }^{*}$ Speaker
} 


\section{Diagonal reflection symmetries}

To start, we show a new set of symmetries [1,2]. The mass matrices of the SM fermions $f=u, d, e$, and neutrinos $v_{L}$ are defined by

$$
\mathcal{L} \ni \sum_{f}-\bar{f}_{L i} m_{f i j}^{B M} f_{R j}-\bar{v}_{L i} m_{v i j}^{B M} v_{L j}^{c}+\text { h.c. . }
$$

These matrices $m_{f}^{B M}$ are assumed to satisfy $\mu-\tau$ reflection symmetries separately [3-5]:

$$
T_{u}\left(m_{u, v}^{B M}\right)^{*} T_{u}=m_{u, v}^{B M}, \quad T_{d}\left(m_{d, e}^{B M}\right)^{*} T_{d}=m_{d, e}^{B M},
$$

where

$$
T_{u}=\left(\begin{array}{ccc}
1 & 0 & 0 \\
0 & 0 & 1 \\
0 & 1 & 0
\end{array}\right), \quad T_{d}=\left(\begin{array}{ccc}
1 & 0 & 0 \\
0 & 0 & -1 \\
0 & -1 & 0
\end{array}\right)
$$

A simultaneous redefinition of all fermion fields $f^{\prime}=U_{B M} f$ and $v^{\prime}=U_{B M} v$ by the following bi-maximal transformation $U_{B M}$,

$$
m_{f} \equiv U_{B M} m_{f}^{B M} U_{B M}^{\dagger}, \quad m_{v} \equiv U_{B M} m_{v}^{B M} U_{B M}^{T}, \quad U_{B M} \equiv\left(\begin{array}{ccc}
1 & 0 & 0 \\
0 & \frac{i}{\sqrt{2}} & \frac{i}{\sqrt{2}} \\
0 & -\frac{1}{\sqrt{2}} & \frac{1}{\sqrt{2}}
\end{array}\right),
$$

leads to the following mass matrices;

$$
m_{u, v}=\left(\begin{array}{ccc}
a_{u, v} & i b_{u, v} & i c_{u, v} \\
i d_{u, v} & e_{u, v} & f_{u, v} \\
i g_{u, v} & h_{u, v} & k_{u, v}
\end{array}\right), m_{d, e}=\left(\begin{array}{ccc}
a_{d, e} & b_{d, e} & c_{d, e} \\
d_{d, e} & e_{d, e} & f_{d, e} \\
g_{d, e} & h_{d, e} & k_{d, e}
\end{array}\right),
$$

with real parameters $a_{f} \sim k_{f}$.

In this basis, the $\mu-\tau$ reflection symmetries (2) are rewritten as

$$
U_{B M} T_{u, d} U_{B M}^{T} m_{u, d}^{*} U_{B M}^{*} T_{u, d} U_{B M}^{\dagger}=m_{u, d}
$$

Surprisingly,

$$
-U_{B M}^{*} T_{u} U_{B M}^{\dagger}=\operatorname{diag}(-1,1,1) \equiv R, \quad U_{B M}^{*} T_{d} U_{B M}^{\dagger}=\operatorname{diag}(1,1,1)=1_{3} .
$$

Then, the $\mu-\tau$ reflection symmetries in the basis are transformed into

$$
R m_{u, v}^{*} R=m_{u, v}, \quad m_{d, e}^{*}=m_{d, e} .
$$

The mass matrices (5) certainly satisfy these conditions. We call such a symmetry diagonal reflection because it is a diagonal remnant of $\mu-\tau$ reflection symmetry after deduction of $\mu-\tau$ symmetry [6]. Each of them is just a generalized $C P$ symmetry [7-10] and no longer a $\mu-\tau$ reflection. 
By combining these symmetries with Hermitian four-zero texture (10) [11], the CKM matrix is reproduced with accuracies of $O\left(10^{-3}\right)$;

$$
V_{\mathrm{CKM}} \simeq\left(\begin{array}{ccc}
1 & \sqrt{m_{u} / m_{c}} & 0 \\
-\sqrt{m_{u} / m_{c}} & 1 & 0 \\
0 & 0 & 1
\end{array}\right)\left(\begin{array}{ccc}
-i & 0 & 0 \\
0 & c_{q} & s_{q} \\
0 & s_{q} & c_{q}
\end{array}\right)\left(\begin{array}{ccc}
1 & -\sqrt{m_{d} / m_{s}} & 0 \\
\sqrt{m_{d} / m_{s}} & 1 & 0 \\
0 & 0 & 1
\end{array}\right),
$$

where $\left|s_{q}\right| \simeq 0.04$ comes from mixings of 23 generations in $M_{u, d}$. This scheme predicts $\left|V_{u b}\right| \simeq$ $\sqrt{m_{u} / m_{c}}\left|V_{c b}\right| \simeq 0.0018$, and it does not match the current observation $\left|V_{u b}^{\text {obs }}\right| \simeq 0.00361$. However, this mismatch can be solved by allowing large 23 elements with small complex phases [12] or by allowing finite $\left(M_{u}\right)_{11} \neq 0$ [2]. The Hermiticity of Yukawa matrices $Y_{u, d, e}$ is justified by the parity symmetry in the left-right symmetric models [13-15].

\section{Universal four-zero texture}

Here, we show the following universal four-zero texture

$$
M_{u, v}=\left(\begin{array}{ccc}
0 & i C_{u, v} & 0 \\
\mp i C_{u, v} & \tilde{B}_{u, v} & B_{u, v} \\
0 & B_{u, v} & A_{u, v}
\end{array}\right), \quad M_{d, e}=\left(\begin{array}{ccc}
0 & C_{d, e} & 0 \\
C_{d, e} & \tilde{B}_{d, e} & B_{d, e} \\
0 & B_{d, e} & A_{d, e}
\end{array}\right)
$$

is compatible with neutrino mixing parameters. The plus (minus) sign in $\mp$ corresponds to a symmetric matrix of neutrinos (a Hermitian matrix of up-type quarks). Since this system has only eight degrees of freedom, the following observables determine the mass matrices; three charged lepton masses at mass of $Z$ boson $m_{Z}$ [16],

$$
m_{e}=486.570 \mathrm{keV}, \quad m_{\mu}=102.718 \mathrm{MeV}, \quad m_{\tau}=1746.17 \mathrm{MeV},
$$

the mixing angles and mass differences of the latest global fit [17]

$$
\begin{aligned}
\theta_{23}^{P D G} & =49.7^{\circ}, \quad \theta_{12}^{P D G}=33.82^{\circ}, \quad \theta_{13}^{P D G}=8.61^{\circ}, \\
\Delta m_{21}^{2} & =73.9 \mathrm{meV}^{2}, \quad \Delta m_{31}^{2}=2525 \mathrm{meV}^{2} .
\end{aligned}
$$

Thus, the remaining four parameters in the neutrino sector, namely the three $C P$ phases $\delta, \alpha_{2,3}$ and the lightest neutrino mass $m_{1}$ are predicted.

The Jarlskog invariant [18] determines the Dirac phase $\delta_{C P}$ as

$$
\sin \delta_{C P}=-0.390, \quad \delta_{C P} \simeq 203^{\circ} .
$$

This is very close to the best fit for the normal hierarchy (NH) $\delta_{C P} /{ }^{\circ}=217_{-28}^{+40}[17]$.

The Majorana phases are calculated from similar rephasing invariants [19]

$$
\begin{aligned}
& I_{1}=\operatorname{Im}\left[\left(U_{\mathrm{MNS}}\right)_{12}^{2}\left(U_{\mathrm{MNS}}\right)_{11}^{* 2}\right]=\frac{1}{4} \sin ^{2} 2 \theta_{12}^{P D G} \cos ^{4} \theta_{13}^{P D G} \sin \alpha_{2}, \\
& I_{2}=\operatorname{Im}\left[\left(U_{\mathrm{MNS}}\right)_{13}^{2}\left(U_{\mathrm{MNS}}\right)_{11}^{* 2}\right]=\frac{1}{4} \sin ^{2} 2 \theta_{13}^{P D G} \cos ^{2} \theta_{12}^{P D G} \sin \alpha_{3}^{\prime},
\end{aligned}
$$


where $\alpha_{3}^{\prime} \equiv \alpha_{3}-2 \delta_{C P}$. A reconstructed mixing matrix $U_{\text {MNS }}$ yields the following results;

$$
\alpha_{2}^{0} \simeq 11.3^{\circ}, \quad \alpha_{3}^{0} \simeq 7.54^{\circ} .
$$

Meanwhile, the original $\mu-\tau$ reflection symmetry restrict the Majorana phases to be $\alpha_{2,3} / 2=n \pi / 2$ $(n=0,1)$ [20]. The nontrivial phase $\pi / 2$ comes from negative masses (after a real rotation). We parameterize these effects as

$$
m_{2}=e^{i \beta_{2}}\left|m_{2}\right|, \quad m_{3}=e^{i \beta_{3}}\left|m_{3}\right|, \quad \beta_{2,3}=0 \text { or } \pi .
$$

The whole Majorana phases are found to be

$$
\left(\alpha_{2}, \alpha_{3}\right)=\left(\alpha_{2}^{0}+\beta_{2}, \alpha_{3}^{0}+\beta_{3}\right)=\left(11.3^{\circ} \text { or } 191.3^{\circ}, 7.54^{\circ} \text { or } 187.54^{\circ}\right) .
$$

Finally, The numerical values of the lightest mass $m_{1}$ are found to be

$$
\begin{aligned}
m_{1} & =6.20 \mathrm{meV} \text { for }\left(\beta_{2}, \beta_{3}\right)=(0,0) \text { or }(\pi, \pi), \\
& =2.54 \mathrm{meV} \text { for }\left(\beta_{2}, \beta_{3}\right)=(0, \pi) \text { or }(\pi, 0),
\end{aligned}
$$

for the $\mathrm{NH}$ case. For the inverted mass hierarchy, the solutions do not have real values and thus contradict the diagonal reflection.

\subsection{Right-handed neutrino mass $M_{R}$}

The right-handed neutrino mass matrix $M_{R}$ can be reconstructed from the type-I seesaw mechanism [21-24] with some GUT relations. For example, a simple $u-v$ unification as realized in Pati-Salam GUT [13] determines $Y_{v}$;

$$
Y_{v}=Y_{u} \simeq \frac{0.9 m_{t} \sqrt{2}}{v}\left(\begin{array}{ccc}
0 & 0.0002 i & 0 \\
-0.0002 i & 0.10 & 0.31 \\
0 & 0.31 & 1
\end{array}\right)
$$

Here, the value of $Y_{u}$ is taken from one of the recent best fits [12].

From the type-I seesaw mechanism and Eq. (22), $M_{R}$ also displays a four-zero texture because the four-zero texture is seesaw invariant $[25,26]$,

$$
M_{R}=\frac{v^{2}}{2} Y_{v} M_{v}^{-1} Y_{v}^{T}=\left(\begin{array}{ccc}
0 & -1.08 i \times 10^{8} & 0 \\
-1.08 i \times 10^{8} & 1.26 \times 10^{14} & 4.07 \times 10^{14} \\
0 & 4.07 \times 10^{14} & 1.32 \times 10^{15}
\end{array}\right) \mathrm{GeV}
$$

Evidently, $M_{R}$ also satisfies diagonal reflection symmetry (8),

$$
R M_{R}^{*} R=M_{R}
$$

Therefore, all the fermion masses respect the diagonal reflection symmetry with the four-zero textures.

The masses of $M_{R}$ are found to be

$$
\left(M_{R 1}, M_{R 2}, M_{R 3}\right)=\left(2.86 \times 10^{6}, 3.73 \times 10^{9}, 1.44 \times 10^{15}\right) \mathrm{GeV} .
$$


This is just an example calculation because it depends on unification schemes. Also, the Yukawa matrix $Y_{v}$ (22) is evaluated at $m_{Z}$ scale. Renormalized values of quark masses at a GUT scale will lead to $O(10)$ smaller masses of $M_{R}$.

Moreover, these symmetries are almost renormalization invariant and realized by vevs of scalar fields $\left\langle\theta_{u}\right\rangle=i v_{u}$ and $\left\langle\theta_{d}\right\rangle=v_{d}$ that only couple to the first generations of SM fermions. Detailed discussions are found in the original papers [1,2].

\section{Acknowledgment}

This study is financially supported by JSPS Grants-in-Aid for Scientific Research No. JP18H01210, No. 20K14459, and MEXT KAKENHI Grant No. JP18H05543.

\section{References}

[1] M.J.S. Yang, Diagonal reflection symmetries and universal four-zero texture, Chin. Phys. C 45 (2021) 043103 [2003. 11701].

[2] M.J.S. Yang, Almost exact diagonal reflection symmetries and three-zero texture, Nucl. Phys. B 972 (2021) 115549 [2103.12289].

[3] P.F. Harrison and W.G. Scott, mu - tau reflection symmetry in lepton mixing and neutrino oscillations, Phys. Lett. B547 (2002) 219 [hep-ph/0210197].

[4] W. Grimus and L. Lavoura, A Nonstandard CP transformation leading to maximal atmospheric neutrino mixing, Phys. Lett. B579 (2004) 113 [hep-ph/0305309].

[5] M.J.S. Yang, Interplay between $\mu-\tau$ reflection symmetry, four-zero texture and universal texture, Phys. Lett. B 806 (2020) 135483 [2002.09152].

[6] T. Fukuyama and H. Nishiura, Mass matrix of Majorana neutrinos, hep-ph/9702253.

[7] G. Ecker, W. Grimus and H. Neufeld, Spontaneous CP Violation in Left-right Symmetric Gauge Theories, Nucl. Phys. B 247 (1984) 70.

[8] M. Gronau and R.N. Mohapatra, Maximal Spontaneous CP Violation and Left-right Symmetry, Phys. Lett. B 168 (1986) 248.

[9] F. Feruglio, C. Hagedorn and R. Ziegler, Lepton Mixing Parameters from Discrete and CP Symmetries, JHEP 07 (2013) 027 [1211 . 5560].

[10] M. Holthausen, M. Lindner and M.A. Schmidt, CP and Discrete Flavour Symmetries, JHEP 04 (2013) 122 [1211.6953].

[11] H. Fritzsch and Z.-z. Xing, A Symmetry pattern of maximal CP violation and a determination of the unitarity triangle, Phys. Lett. B353 (1995) 114 [hep-ph/9502297].

[12] Z.-z. Xing and Z.-h. Zhao, On the four-zero texture of quark mass matrices and its stability, Nucl. Phys. B 897 (2015) 302 [1501.06346]. 
[13] J.C. Pati and A. Salam, Lepton Number as the Fourth Color, Phys. Rev. D10 (1974) 275.

[14] G. Senjanovic and R.N. Mohapatra, Exact Left-Right Symmetry and Spontaneous Violation of Parity, Phys. Rev. D 12 (1975) 1502.

[15] R.N. Mohapatra and J.C. Pati, Left-Right Gauge Symmetry and an Isoconjugate Model of CP Violation, Phys.Rev. D11 (1975) 566.

[16] Z.-z. Xing, H. Zhang and S. Zhou, Impacts of the Higgs mass on vacuum stability, running fermion masses and two-body Higgs decays, Phys. Rev. D 86 (2012) 013013 [1112 . 3112 ].

[17] I. Esteban, M.C. Gonzalez-Garcia, A. Hernandez-Cabezudo, M. Maltoni and T. Schwetz, Global analysis of three-flavour neutrino oscillations: synergies and tensions in the determination of $\theta_{23}, \delta_{C P}$, and the mass ordering, JHEP 01 (2019) 106 [1811.05487].

[18] C. Jarlskog, Commutator of the Quark Mass Matrices in the Standard Electroweak Model and a Measure of Maximal CP Violation, Phys. Rev. Lett. 55 (1985) 1039.

[19] J.F. Nieves and P.B. Pal, Minimal Rephasing Invariant CP Violating Parameters With Dirac and Majorana Fermions, Phys. Rev. D 36 (1987) 315.

[20] Z.-z. Xing and J.-y. Zhu, Neutrino mass ordering and $\mu-\tau$ reflection symmetry breaking, Chin. Phys. C41 (2017) 123103 [1707.03676].

[21] P. Minkowski, $\mu \rightarrow$ er at a Rate of One Out of $10^{9}$ Muon Decays?, Phys. Lett. 67B (1977) 421.

[22] T. Yanagida, Horizontal gauge symmetry and masses of neutrinos, Conf. Proc. C7902131 (1979) 95.

[23] R.N. Mohapatra and G. Senjanovic, Neutrino Mass and Spontaneous Parity Nonconservation, Phys. Rev. Lett. 44 (1980) 912.

[24] M. Gell-Mann, P. Ramond and R. Slansky, Complex spinors and unified theories, Conf. Proc. C790927 (1979) 315.

[25] H. Nishiura, K. Matsuda and T. Fukuyama, Lepton and quark mass matrices, Phys. Rev. D60 (1999) 013006 [hep-ph/9902385].

[26] H. Fritzsch and Z.-z. Xing, Mass and flavor mixing schemes of quarks and leptons, Prog. Part. Nucl. Phys. 45 (2000) 1 [hep-ph/9912358]. 